\title{
Essais préliminaires d'inséminations artificielles en station chez les femelles zébus Azaouak au Niger
}

\author{
M. Issa ${ }^{1 *}$ H. Marichatou ${ }^{2}$ C. Semita ${ }^{3}$ M. Bouréima ${ }^{4}$ \\ M. Kéita ${ }^{4}$ T. Nervo ${ }^{3}$ A. Yénikoye ${ }^{2}$ F. Cristofori ${ }^{3}$ \\ G. Trucchi ${ }^{3}$ G. Quaranta ${ }^{3}$
}

Mots-clés

Bos indicus - Vache Azaouak Insémination artificielle - Cycle œstral - Niger.

\begin{abstract}
Résumé
Des inséminations artificielles sur chaleurs induites et chaleurs naturelles ont été effectuées sur 138 femelles zébus Azaouak à la Station sahélienne expérimentale de Toukounous. Les chaleurs ont été induites par I'utilisation de l'implant Crestar, de la spirale vaginale, et d'injections de prostaglandine et de gonadolibérine $(\mathrm{GnRH})$. Deux inséminations ont été faites : la première à l'observation des chaleurs et la seconde 12 heures après. L'influence de paramètres zootechniques a été testée. Les taux non cumulés de gravidité obtenus ont été de 24,5 p. 100 à la première insémination, 33,72 p. 100 à la deuxième, et 29,48 p. 100 à la troisième et au-delà. L'utilisation de semence fraîche, et l'utilisation de vaches et non de génisses ont fait partie des paramètres zootechniques influençant significativement et positivement les inséminations. Le délai de 60 jours post partum généralement observé avant les inséminations n'a pas eu d'effet significatif sur les résultats. La saison d'insémination n'ayant pas influencé les résultats, des mises bas ont pu être observées tout au long de l'année. L'état nutritionnel a par ailleurs été un facteur essentiel de réussite des inséminations ; les résultats ont traduit la capacité des zébus Azaouak à valoriser les fourrages en toute saison, avec une reprise rapide de l'activité ovarienne après la mise bas.
\end{abstract}

\section{INTRODUCTION}

Au Niger l'élevage constitue un élément important du secteur agricole avec 12 p. 100 de participation au PIB (18). Le cheptel nigérien a été estimé en 2007 à près de 31036040 mammifères domestiques toutes espèces confondues (14), représentant une valeur totale de 706 milliards de franc CFA et une valeur annuelle de production de 191,5 milliards de FCFA (18).

L'élevage concerne 95 p. 100 de la population dont 20 p. 100 en tirent l'essentiel de leur subsistance. Cependant, le développe-

\footnotetext{
1. Faculté des Sciences, Université Abdou Moumouni, Niamey, Niger. 2. Faculté d'Agronomie, Université Abdou Moumouni, Niamey, Niger.

3. Dipartimento di Patologia animale, Università di Torino, Italia.

4. IPR, Bamako, Mali.

* Auteur pour la correspondance

Tél. : +22793930350/+22796656756

Fax : +22720315862

E-mail : mougarbeye@yahoo.fr
}

ment de l'élevage est confronté à une contrainte majeure, liée à la productivité du cheptel bovin, estimé à 7336088 d'individus en 2007 (14). Avec la croissance démographique, la production laitière nationale ne satisfait plus la demande, ce qui oblige le Niger à importer annuellement des produits laitiers pour une valeur de 6,6 milliards de FCFA. Cela s'explique par un potentiel génétique faible avec des performances laitières très limitées. Pour remédier à cette situation, l'Etat nigérien avait opté pour l'amélioration du potentiel génétique des races locales. C'est ainsi que la race Azaouak, considérée comme la race bovine la mieux adaptée aux conditions environnementales sahéliennes, a été retenue. Un programme de sélection de cette race a été mis en place à la Station sahélienne expérimentale de Toukounous depuis 1954. Cette sélection a abouti à une caractérisation phénotypique de la race, avec une légère amélioration de la production de lait.

Cette situation rend nécessaire d'introduire les biotechnologies de la reproduction, en l'occurrence l'insémination artificielle qui, d'une part, facilitera la diffusion des potentialités de la race 
Azaouak, et, d'autre part, permettra d'accroître rapidement les productions bovines par le croisement des races locales avec des races exotiques.

La pratique de l'insémination artificielle nécessite la prise en compte des principaux facteurs susceptibles d'influencer la fertilité des femelles. La présente étude rapporte les premiers résultats d'inséminations artificielles au Niger et contribue à une meilleure connaissance de la fertilité des femelles Azaouak après insémination, induction des chaleurs et chaleurs naturelles.

\section{MATERIEL ET METHODES}

L'étude a été menée en milieu réel de 2002 à 2008 à la Station sahélienne expérimentale de Toukounous (SSET), station à vocation laitière, située à $200 \mathrm{~km}$ de Niamey $\left(14^{\circ} 31^{\prime}\right.$ de lat. $\mathrm{N}$ et $3^{\circ} 18^{\prime}$ de long. E). Le climat est de type sahélien avec une pluviométrie moyenne de $300 \mathrm{~mm}$ par an, une température moyenne de $34{ }^{\circ} \mathrm{C}$ avec des minima de 10 à $20{ }^{\circ} \mathrm{C}$ en décembre et janvier, et des maxima de 40 à $45^{\circ} \mathrm{C}$ en mai et juin.

Le système d'élevage était de type extensif avec un minimum d'intrants. Les animaux (au nombre de 1000 environ) étaient répartis selon l'âge, le sexe et l'état physiologique (lactation, gravidité) sur une superficie de 4400 ha, dans des aires de pâturage clôturées par du grillage. Les troupeaux vivaient toute l'année sur des parcelles bien délimitées. L'alimentation était entièrement basée sur le pâturage naturel. Seules les femelles en lactation recevaient une complémentation en graines de coton. Tous les animaux étaient suivis sur le plan sanitaire.

Les femelles destinées aux essais d'insémination artificielle (20 à 30 femelles en rotation) étaient issues du troupeau de base de la station. Elles étaient gardées, isolées des mâles, sur une ou deux parcelles non loin du laboratoire. Leur alimentation était entièrement basée sur parcours naturel. Elles étaient conduites au laboratoire uniquement pour les besoins de synchronisation, d'insémination et de diagnostic de gravidité. Une fois l'activité terminée, elles étaient ramenées sur leurs parcelles. Par ailleurs, après confirmation de la gravidité, elles étaient conduites dans le troupeau des vaches gravides de la station et remplacées par d'autres vides.

Des femelles ont parfois été retournées au laboratoire après la mise bas pour un deuxième ou un troisième séjour. Elles ont alors été considérées comme nouvelles (en gardant le même numéro d'identification) et soumises à une nouvelle série de synchronisation et d'inséminations.

Malgré le système de sécurité mis en place pour séparer les femelles des mâles, il a été enregistré quelques fois des saillies naturelles. Ces femelles saillies ont été retirées du troupeau expérimental à compter de la date de saillie.

\section{Méthodes d'induction des chaleurs}

Au total 149 femelles qui provenaient toutes de la SSET ont été utilisées. Cependant, une femelle pouvait être l'objet de plusieurs synchronisations.

\section{Implant}

L'implant Crestar a été utilisé comme méthode de contrôle de l'œstrus suivant le chronogramme suivant : J0, pose de l'implant Crestar à l'oreille ; J7, injection en intramusculaire (IM) de $2 \mathrm{ml}$ de PGF2 $\alpha$ (500 $\mu \mathrm{g}$ Cloprostenol) ; J9, retrait de l'implant avec injection de 350 UI de gonadotrophine de sérum de jument gravide (PMSG ; Folligon) ; J11, détection des chaleurs à partir de cette date.

\section{Spirale vaginale}

La spirale vaginale progesterone releasing intra vaginal device (PRID ; Ceva) a été utilisée comme deuxième méthode de contrôle de l'œstrus. Le protocole d'induction a été le suivant : J0, insertion vaginale du PRID ; J7, injection en IM de $2 \mathrm{ml}$ de PGF2 $\alpha$ (500 $\mu \mathrm{g}$ Cloprostenol) ; J9, retrait du PRID avec injection en intramusculaire de 350 UI de PMSG (Folligon); J11, détection des chaleurs à partir de cette date.

\section{Injections de prostaglandine}

La méthode classique des deux injections de prostaglandine pour faire régresser les corps jaunes fonctionnels et induire la survenue des chaleurs n'a pas été utilisée. Une seule injection de $2 \mathrm{ml}$ de prostaglandine a été effectuée, soit six à sept jours après le début des chaleurs, ou après détection d'un corps jaune au palper rectal.

\section{Injections de gonadolibérine}

Le protocole avec la gonadolibérine $(\mathrm{GnRH})$ a été réalisé pour synchroniser les ovulations (13) selon le chronogramme suivant : J0, injection en IM de $1 \mathrm{ml}$ de GnRH (100 $\mu \mathrm{g}$ Gonadorelin) ; J7, injection en IM de $2 \mathrm{ml}$ de PGF2 $\alpha$ (500 $\mu \mathrm{g}$ Cloprostenol) ; J9, injection en IM de $1 \mathrm{ml}$ de GnRH (100 $\mu \mathrm{g}$ Gonadorelin).

\section{Détection des chaleurs}

Les chaleurs ont été détectées par observation directe du berger en se basant sur le comportement des femelles suspectées. L'immobilisation pour se laisser monter par ses congénères a été le critère principal de la survenue des chaleurs. Le contrôle des chaleurs a été effectué le matin à $8 \mathrm{~h}$ et le soir vers $16 \mathrm{~h}$.

\section{Inséminations artificielles}

Sur les 149 femelles synchronisées, 11 n'ont pas été inséminées pour des raisons diverses (saillie naturelle, sortie du troupeau, enlèvement de la station...). Sur les 138 femelles inséminées, certaines l'ont été plusieurs fois, d'autres ont effectué plusieurs séjours (une femelle mettant bas suite à une insémination pouvait revenir pour une nouvelle série).

Les inséminations ont été effectuées sur chaleurs observées, mais les femelles traitées au GnRH l'ont été sans détection de chaleurs; dans ce dernier cas, le principe est une synchronisation des ovulations (13).

Deux inséminations successives ont été effectuées : la première au moment de la détection des chaleurs pour les femelles à chaleurs induites, 20 heures après la dernière injection de GnRH, et la deuxième 12 heures après la première. En cas d'inséminations infructueuses, les inséminations ont été renouvelées jusqu'à cinq fois.

\section{Diagnostic de gravidité}

Deux méthodes de diagnostic de gravidité ont été utilisées : l'observation simple des retours de chaleurs et la palpation transrectale trois mois après l'insémination.

\section{Analyse des données}

Les femelles ont été dénombrées en fonction de certains paramètres, notamment la méthode d'induction, la parité, la nature de la semence utilisée à l'insémination, le délai post partum à la synchronisation, et la saison de synchronisation. Pour ce dernier paramètre trois saisons ont été retenues : juillet à octobre, période correspondant à la saison de pluie, avec une bonne alimentation et des amplitudes thermiques peu élevées ; novembre à février, période 
correspondant à la saison sèche et froide, avec une bonne disponibilité en fourrage et de faibles amplitudes thermiques ; et mars à juin, période correspondant à la saison sèche et chaude avec assez souvent une mauvaise disponibilité en fourrage et des amplitudes thermiques élevées. Le test du $\chi^{2}$ au risque $\alpha=5$ p. 100 a été utilisé pour étudier la liaison de ces paramètres avec le taux de réussite à la première insémination.

\section{RESULTATS}

Les taux de réponse aux inductions des chaleurs par les principales méthodes de traitement utilisées sont rapportés dans le tableau I. La GnRH ayant concerné des effectifs réduits ne figure pas dans le tableau. Il y a eu une différence significative entre les réponses aux inductions des chaleurs. L'utilisation du Crestar a permis d'obtenir un taux de réponse plus élevé que les autres méthodes $\left(\chi^{2}=19,7\right.$; $\mathrm{p}=0,00)$.

La répartition de la gravidité en fonction du rang d'insémination est affichée dans le tableau II.

Le tableau III résume les résultats à la première insémination et par traitement de synchronisation.

L'induction de l'œstrus a été significativement meilleure avec l'implant Crestar qu'avec les autres méthodes d'induction $\left(\chi^{2}=19,7\right.$; $\mathrm{p}=0,00)$, mais il n'y a pas eu de différence significative de fertilité selon les méthodes d'induction $\left(\chi^{2}=1,08 ; \mathrm{p}=0,89\right)$.

Le tableau IV montre la répartition de la gravidité en fonction des chaleurs induites ou naturelles. Le taux d'inséminations en chaleurs naturelles (44 p. 100) n'a pas été significativement plus élevé $\left(\chi^{2}=3,71 ; \mathrm{p}=0,054\right)$ que celui des chaleurs induites $(25,28$ p. 100).

Le taux de réussite des inséminations a été significativement plus élevé $\left(\chi^{2}=5,27 ; p=0,02\right)$ avec la semence fraîche. Les chances de réussite ont été réduites de moitié avec la semence congelée (tableau V).

Le taux de réussite à la première insémination a été influencé significativement par la parité de la femelle $\left(\chi^{2}=5,38 ; p=0,02\right)$. Les inséminations ont mieux réussi avec les vaches (tableau VI).

Le résultat n'a pas montré d'influence significative $\left(\chi^{2}=0,005\right.$; $\mathrm{p}=0,95)$ du délai post partum sur le succès des inséminations (tableau VII)

La réussite des inséminations n'a pas été significativement liée aux saisons $\left(\chi^{2}=0,14 ; p=0,93\right)$ (tableau VIII), indiquant une absence probable de saisonnalité de la fertilité des femelles Azaouak, avec pour conséquence des mises bas observées à tout moment de l'année.

\section{Tableau I}

Réponse aux inductions des chaleurs

\begin{tabular}{lccc} 
Traitement & Chaleurs & $\varnothing$ Chaleurs & $\begin{array}{c}\text { Taux de } \\
\text { réponse (\%) }\end{array}$ \\
\hline Crestar & 205 & 55 & 78,84 \\
PRID & 55 & 37 & 59,78 \\
Injection unique & 17 & 16 & 51,51 \\
de prostaglandine & & & \\
Total & 277 & 108 & 71,94
\end{tabular}

Tableau II

Résultats de la gravidité en fonction du rang d'insémination artificielle

\begin{tabular}{lcccc} 
& \multicolumn{4}{c}{ Rang d'insémination artificielle } \\
\cline { 2 - 4 } & $\mathbf{1}^{\text {er }}$ & $\mathbf{2}^{\mathbf{e}}$ & $\mathbf{3}^{\mathbf{e}}$ et plus & Total \\
\hline $\begin{array}{l}\text { Nb. d'actes } \\
\text { d'insémination }\end{array}$ & 151 & 86 & 78 & 315 \\
$\begin{array}{l}\text { Nb. d'animaux } \\
\text { gravides }\end{array}$ & 37 & 29 & 23 & 89 \\
$\begin{array}{l}\text { Taux de gravidité } \\
\text { Taux de }\end{array}$ & 24,5 & 33,72 & 29,48 & 28,25
\end{tabular}

Tableau III

Résultats de la première insémination artificielle par traitements $d^{\prime}$ induction

\begin{tabular}{lrcc} 
Traitement & Nb. d'IA & $\begin{array}{c}\text { Nb. d'animaux } \\
\text { gravides }\end{array}$ & $\begin{array}{c}\text { Gravidité } \\
(\mathbf{\%})\end{array}$ \\
\hline Crestar & 100 & 22 & 22 \\
PRID & 31 & 8 & 25,80 \\
Injection de & 7 & 2 & 28,57 \\
prostaglandine & & & \\
GnRH & 7 & 3 & 42,85 \\
Chaleurs naturelles & 6 & 2 & 33,33 \\
Total & 151 & 37 & 24,5
\end{tabular}

IA : insémination artificielle

\section{Tableau IV}

Toutes les inséminations artificielles en fonction de la nature de l'œstrus

\begin{tabular}{lccc} 
Traitement & Nb. d'IA & $\begin{array}{c}\text { Nb. d'animaux } \\
\text { gravides }\end{array}$ & $\begin{array}{c}\text { Gravidité } \\
(\%)\end{array}$ \\
\hline $\begin{array}{l}\text { IA sur induction } \\
\text { des chaleurs }\end{array}$ & 265 & 67 & 25,28 \\
$\begin{array}{l}\text { IA sur chaleurs } \\
\text { naturelles }\end{array}$ & 50 & 22 & 44 \\
Total & 315 & 89 & 28,25 \\
\hline
\end{tabular}

IA : insémination artificielle

\section{Tableau V}

Gravidité en fonction de la nature de la semence

\begin{tabular}{lccc|} 
Semence & Nb. d'IA & $\begin{array}{c}\text { Nb. d'animaux } \\
\text { gravides }\end{array}$ & $\begin{array}{c}\text { Gravidité } \\
(\%)\end{array}$ \\
\hline Fraîche & 254 & 81 & 31,88 \\
Congelée & 61 & 8 & 13,11 \\
Total & 315 & 89 & 28,25
\end{tabular}

IA : insémination artificielle 
Tableau VI

Parité lors de la première insémination artificielle

$\begin{array}{lccc}\text { Parité } & \text { Gravide } & \text { Nb. d'IA } & \text { Gravidité (\%) } \\ \text { Génisse } & 3 & 39 & 7,69 \\ \text { Vache } & 34 & 112 & 30,35 \\ \text { Total } & 37 & 151 & 24,5\end{array}$

IA : insémination artificielle

\section{Tableau VII}

Résultats des inséminations artificielles en fonction du post partum

\begin{tabular}{lccc} 
& Gravide & Nb. d'IA & Total (\%) \\
\hline Post partum $<60$ j & 8 & 27 & 29,62 \\
Post partum $\geq 60$ j & 26 & 85 & 30,58 \\
Total & 34 & 112 & 30,35
\end{tabular}

IA : insémination artificielle

\section{Tableau VIII}

Inséminations artificielles en fonction de la saison à la première insémination

$\begin{array}{lccc}\text { Saison } & \text { Gravide } & \text { Nb. d'IA } & \text { Total (\%) } \\ \text { Mars à juin } & 9 & 36 & 25 \\ \text { Juillet à octobre } & 13 & 49 & 26,53 \\ \text { Novembre à février } & 15 & 66 & 23,07 \\ \text { Total } & 37 & 151 & 24,5\end{array}$

IA : insémination artificielle

\section{DISCUSSION}

Des trois méthodes de synchronisation, seule l'implant Crestar a donné un résultat nettement supérieur à la moyenne (78,84 p. 100), un niveau de réponse proche de celui par exemple des femelles Baoulé (80 p. 100) (2). Les réponses du zébu Azaouak au PRID (59,78 p. 100) et aux prostaglandines (51,51 p. 100) ont été faibles par rapport à celles observées chez les zébus Bunaji (78,3 p. 100) (19) et Bokolodji, (64,3 p. 100) (17). Ceci était probablement lié, d'une part, à une imprécision dans la détection du corps jaune au palper rectal ou à une insuffisance de la dose de prostaglandine utilisée $(17,19)$, et, d'autre part, à des facteurs tels que la race, le poids et la parité (19).

Le taux de gravidité de 33,72 p. 100 à la deuxième insémination, obtenu en milieu sahélien difficile, a été sensiblement meilleur que le taux global enregistré sur plusieurs races au Mali (31,50 p. 100), dans les mêmes conditions climatiques, après deux inséminations (7). En milieu européen, Fréret et coll. (6) rapportent un taux de 45,9 p. 100 en première insémination chez la race Holstein. La dose de 350 UI de PMSG utilisée pour accentuer la réponse des femelles a été probablement faible par rapport à celle utilisée par d'autres auteurs sur des races africaines $(500$ à 800 UI) $(2,12)$. Il est donc possible d'améliorer ces taux en augmentant la dose audelà de 350 UI.

Au cours de cet essai, le mode d'induction de l'œstrus n'a pas significativement affecté le taux de réussite des premières inséminations comme l'ont montré d'autres auteurs sur des génisses zébus (3). Cependant, dans la présente étude, les effectifs des traitements de prostaglandine et de $\mathrm{GnRH}$ ont été relativement faibles, masquant probablement un effet significatif entre les modes de traitement. Le système Ovsynch (13), qui utilise de la GnRH pour synchroniser les ovulations, serait probablement le moyen le plus efficace pour entraîner la gravidité.

Les résultats ont montré que le taux de gravidité des chaleurs naturelles (44 p. 100) n'a pas été significativement différent de celui des chaleurs induites $(25,28$ p. 100). En revanche, il y a eu une différence significative entre le taux de gravidité en semence fraîche (31,88 p. 100) et en semence congelée (13,11 p. 100). Les ovocytes issus des œstrus naturels ont été beaucoup plus fertiles que ceux ayant évolué après induction. Les probabilités d'une fécondation ont été plus élevées avec une semence fraîche ayant une bonne motilité et une viabilité qui pouvait durer plusieurs heures (4) qu'avec une semence congelée à la vapeur d'azote liquide dans un bac en polystyrène qui a une faible viabilité.

A Toukounous, la parité a aussi joué un rôle significatif dans le succès des inséminations. La gravidité après induction de l'œstrus a été obtenue plus fréquemment chez les vaches $(30,35$ p. 100) que chez les génisses (7,69 p. 100). Les génisses étaient probablement non cycliques ou impubères lors des traitements d'induction de l'œstrus. Le seul critère de $230 \mathrm{~kg}$ pour la mise en reproduction des génisses dans la station (9) était probablement insuffisant ; il fallait en plus du poids observer les premières chaleurs avant de les destiner à un schéma d'insémination artificielle. Le taux de réponse à l'induction des chaleurs a été plus élevé chez les vaches [77 p. 100 (primipares) et 70 p. 100 (multipares)] que chez les génisses (57,5 p. 100). D'autres auteurs ont signalé un effet de la parité sur la fertilité qui est plus important chez les multipares (48 p. 100) que chez les primipares $(37$ p. 100) $(15,16)$. Ceci pouvait s'expliquer en partie par les taux de cyclicité avant traitement (3).

Le délai post partum et la saison de synchronisation n'ont pas affecté significativement la réussite des inséminations. Ainsi les femelles ont repris leur cyclicité et ont été aptes à une fécondation sans attendre le délai critique de 60 jours post partum, quelle qu'ait été la saison et lorsqu'il n'y avait pas d'infection des voies génitales, ce qui pouvait empêcher l'état gravide (5). Les travaux de Grimard et coll. (8), et Humblot et coll. (10) ont montré des écarts de fertilité atteignant 15 à 30 points entre les femelles à vêlage sans assistance et celles ayant subi une extraction forcée ou une césarienne. Pour le délai post partum, certains auteurs ont obtenu des taux plus élevés que ceux de la présente étude : 41 p. 100 (post partum > 76 jours) (16), 49,2 p. 100 (10) et 72 p. 100 (5) (post partum > 70jours), contre 30,58 p. 100 (post partum $>60$ jours) dans cette étude.

L'absence d'influence des saisons sur la réussite des inséminations a traduit la rusticité de la race Azaouak qui s'adapte à toutes les situations saisonnières. Ce résultat a également confirmé la répartition des vêlages issus des montes naturelles au niveau de la station (1). Cette répartition est à peu près homogène tout au long de l'année depuis plus de 30 ans. Les auteurs ont conclu que le déficit fourrager n'a pas eu d'impact sur les performances de reproduction, car même les années de grandes sécheresses de 1987 (141 mm) et $1990(231 \mathrm{~mm})$ n'ont pas eu d'influence sur les performances de reproduction à la station. 


\section{CONCLUSION}

Les résultats de la présente étude ont montré une meilleure réponse à l'induction de l'œstrus avec l'utilisation du Crestar chez la race Azaouak. Après trois inséminations, un taux de gravidité de 29,48 p. 100 a pu être obtenu. Il y a tout de même eu une baisse de la fertilité par rapport au taux de la deuxième insémination $(33,72$ p. 100). Le taux de gravidité de la première insémination pourrait être amélioré en élevant la dose de PMSG. Le taux de gravidité n'a pas dépendu du type d'induction de l'œstrus.
Parmi les paramètres zootechniques, l'utilisation de vaches et non pas de génisses, et l'utilisation de semence fraîche ont influencé positivement le succès des inséminations. Les vaches se sont rapidement remises à la reproduction et ont été aptes à la fécondation sans observation d'un délai de 60 jours post partum. La saison d'insémination n'a pas non plus influencé le résultat des inséminations. Ceci traduit la capacité d'adaptation de cette race au milieu sahélien et à valoriser le fourrage, même pendant les périodes de sécheresse.

\section{BIBLIOGRAPHIE}

1. ACHARD F., CHANONO M., 1997. Mortalité et performances de reproduction chez le zébu Azaouak à la station de Toukounous, Niger (1986-1992). Revue Elev. Méd. vét. Pays trop., 50 : 325-333.

2. CHICOTEAU P., CLOE L., BASSINGA A., 1986. Essais préliminaires de synchronisation des chaleurs chez la femelle Baoulé. Revue Elev. Méd. vét. Pays trop., 39 : 161-163.

3. CORBET N.J., MILLER R.G., BINDON B.M., BURROW H.M., D)OCCHIO M.J., ENTWISTLE K.W., FITZPATRICK L.A., WILKINS J.F., KINDER J.E., 1999. Synchronization of estrus and fertility in zebu beef heifers treated with three estrus synchronization protocols. Theriogenology, 51: 647-659.

4. CRISTOFORI F., ISSA M., YENIKOYE A., TRUCCHI G., QUARANTA G., CHANONO M., SEMITA C., MARICHATOU H., MATTONI M., 2005. Artificial insemination using local cattle breeds in Niger. Trop. Anim. Health Prod., 37: 167-172.

5. FOURNIER R., DRIANCOURT M.-A., 2007. Maitrise de l'œstrus en troupeau allaitant dans le contexte européen. Angers, France, Intervet, $9 \mathrm{p}$.

6. FRERET S., PONSART C., RAID., JEAN GUYOT N., PACCARD P., HUMBLOT P., 2006. Facteurs de variation en première insémination artificielle et taux de mortalités embryonnaires en élevage laitier Prim' Holstein. In : $13^{\text {es }}$ Rencontres Recherches Ruminants, Paris, France.

7. GADOT GARBA BALKISSA, 2007. Maitrise du cycle chez les bovins dans les élevages périurbains de Bamako et zone rurale : détermination du degré d'efficacité des traitements à base de progestagènes. Mém. fin de cycle, IPR/IFRA, Katibougou, Mali, 41 p.

8. GRIMARD B., NUMBLOT P., PONTER R.A., CHASTANT S, CONSTANT F., MIALOT J.-P., 2003. Efficacité des traitements de synchronisation des chaleurs chez les bovins. Prod. Anim., 16:211, 227. 9. GUY-MICHEL B., 2000. Le zébu Azawak, un label à la peine. Echos Sahel (5).

10. HUMBLOT P, GRIMARD B, RIBON O, KHIREDDNE B, DERVISHI V., THIBIER M.,1996. Sources of variation of postpartum cyclicity, ovulation and pregnancy rates in primiparous Charolais cows treated with Norgestonet implant and PNSG. Theriogenology, 46: 1086-1096.
11. Le Niger et ses économies. htt://fr.encarta.msn.Com/ encyclopedia_761577598/Niger.html

12. LHOSTE P., PIERSON J., 1976. L'expérimentation de l'insémination au Cameroun, par importation de semence congelée. II. Essai de synchronisation de l'œstrus sur femelles zébus. Revue Elev. Méd. vét. Pays trop., 29 : 67-74.

13. MIALOT J.P., CONSTANT F., DEZAUX P., GRIMARD B., DELETAN G.F., PONTER A.A., 2003. Estrus synchronization in beef cows: comparaison between GnRH $+\mathrm{PGF}_{2 \alpha}+\mathrm{GnRH}$ and PRID $+\mathrm{PGF}_{2 \alpha}+$ eCG. Theriogenology, 60: 319-330.

14. MINISTERE DES RESSOURCES ANIMALES, 2007. Recensement général de l'agriculture et du cheptel. Niamey, Niger, ministère des Ressources animales.

15. PONSART C., SANAA M., HUMBLOT P., GRIMARD B., JEANGUYOT N., POTER A.A., VIEL J.P., 1996. Variation factors of pregnancy rates after estrus synchronization treatment in French Charolais beef cows. Vet. Res., 27: 227-239.

16. PURSLEY J.R., KOSOROK M.R., WILTBANK M.C., 1997. Reproductive management of lactating dairy cows using synchronization of ovulation. J. Dairy Sci., 80: 301-306.

17. REKWOT P.I., OYEDIPE E.O., MUKASA-MUGERWA E., SEKONI V.O., AKINPELUMI O.P., ANYAM A.A., 1999. Fertility in zébu cattle (Bos indicus) after prostaglandin administration and artificial insemination. Vet. J., 158: 53-58.

18. STRATEGIE DE DEVELOPPEMENT RURAL, 2006. http://www. strategie-developpement-rural-niger.org

19. VOH A.A., OGWU D., OYEDIPE E.O., 2004. Fertility of Bunaji (zebu) cows after treatment with PRID with or without PGF2 $\alpha$. Trop. Anim. Health Prod., 36: 483-497.

Accepté le 29.03.2011 


\section{Summary}

Issa M., Marichatou H., Semita C., Bouréima M., Kéita M., Nervo T., Yénikoye A., Cristofori F., Trucchi G., Quaranta G. Preliminary trials of artificial insemination in Azawak zebu females in station in Niger

Artificial inseminations of estrus-induced and natural estrus were carried out in 138 Azawak zebu females at the Sahelian Experimental Station of Toukounous. Estrous was induced with a Crestar implant, a progesterone releasing intra-vaginal device, and prostaglandin and gonadotropin-releasing hormone $(\mathrm{GnRH})$ injections. Two inseminations were performed: the first one at estrus observation and the second one 12 hours later. The influence of zootechnical parameters was tested. The achieved non cumulative pregnancy rates were $24.5 \%$ at first insemination, $33.72 \%$ at second, and $29.48 \%$ at third and beyond. The use of fresh semen and of cows instead of heifers was among the zootechnical parameters that significantly and positively influenced inseminations. The 60-day postpartum period usually observed before insemination had no significant effect on the results. As the season of insemination did not influence the results, calvings were observed throughout the year. The nutritional status was also an essential factor of successful inseminations. The results highlighted the ability of Azawak zebus to make optimum use of fodder in all seasons, with ovarian activity resuming soon after parturition.

Keywords: Bos indicus - Azawak cow - Artificial insemination - Estrus cycle - Niger.

\section{Resumen}

Issa M., Marichatou H., Semita C., Bouréima M., Kéita M., Nervo T., Yénikoye A., Cristofori F., Trucchi G., Quaranta G. Ensayos preliminares de inseminaciones artificiales en estación en hembras cebúes Azawak en Níger

Se efectuaron inseminaciones artificiales en calores inducidos y calores espontáneos, en 138 hembras cebú Azawak en la estación sahelina experimental de Toukounous. Los calores fueron inducidos mediante la utilización de implantes Crestar, de la espiral vaginal y de inyecciones de prostaglandina y de gonadoliberina $(\mathrm{GnRH})$. Se hicieron dos inseminaciones: la primera a la observación de los calores y la segunda 12 horas después. Se estudió la influencia de los parámetros zootécnicos. Las tasas no acumulativas de gravedad obtenidas fueron de $24,5 \%$ a la primera inseminación, $33,72 \%$ a la segunda y $29,48 \%$ a la tercera y más allá. El uso de semen fresco y el uso de vacas y no de novillas fueron parte de los parámetros zootécnicos que influenciaron significativamente y positivamente las inseminaciones. El periodo de 60 días post partum generalmente respetado antes de las inseminaciones no tuvo efecto significativo en los resultados. La época de inseminación no influenció los resultados, se observaron partos durante todo el año. El estado nutricional fue por otro lado un factor esencial de éxito de las inseminaciones; los resultados tradujeron la capacidad de los cebúes Azawak para valorizar los forrajes en toda época, con una toma rápida de la actividad ovárica después del parto.

Palabras clave: Bos indicus - Vaca Azawak - Inseminación artificial - Ciclo estral - Níger. 